\title{
Arthritis, progressive maculopapular rash and severe peripheral neuropathy in a 60-year-old man
}

\author{
François Tremblay MD, Martin Albert MD
}

See also the practice article by Vázquez-López and Gonzalez-Lara on page 211 and at www.cmaj.ca/lookup/doi/10.1503/cmaj.130221

Competing interests: None declared.

This article has been peer reviewed.

\section{Correspondence to:}

Martin Albert,

m.albert@umontreal.ca

CMAJ 2014. DOI:10.1503/ cmaj.130688
A 60-year-old man born in Canada was referred to our clinic because of arthritis, a progressive maculopapular rash and severe peripheral neuropathy. He had a history of hypothyroidism and hypogonadism, completely corrected with levothyroxine and testosterone, respectively. He had been evaluated five years earlier for symmetrical arthritis of the small joints in both hands that was associated with morning stiffness. At the time, his rheumatoid factor and cyclic citrullinated peptide antibody (anti-CCP) test results were negative. Seronegative rheumatoid arthritis had been diagnosed and oral treatment with prednisone and methotrexate started. The patient had stopped the methotrexate because of lack of effect.

A few months after the start of the immunosuppressive agents, a progressive erythematous maculopapular rash had developed on the patient's face, trunk and limbs. A skin biopsy had shown the presence of granulomas. Interstitial granulomatous dermatitis associated with rheumatoid disease had been diagnosed and topical glucocorticoid treatment prescribed. However, the rash continued to progress slowly over the years.

About one year after the initial presentation and six months before referral to our clinic, the patient reported edema of his limbs and glove-stocking sensory loss associated with burning pain. The neurologic symptoms were disabling and kept him from working as a car mechanic. A neurologist evaluated the patient and performed electromyography, which showed a mixed peripheral neuropathy. All investigations to elucidate the cause of the neuropathy were negative, and the neurologist diagnosed familial sensitivomotor polyneuropathy. The patient was subsequently referred to our clinic by his family physician for further investigation.

On physical examination, he had no fever. $\mathrm{He}$ had a confluent erythematous, maculopapular, hypoesthetic rash on his face, trunk and limbs that spared the warmer areas of the body (Figure 1). Other dermatologic findings included thickening of the skin over his nose and loss of his eyebrows.

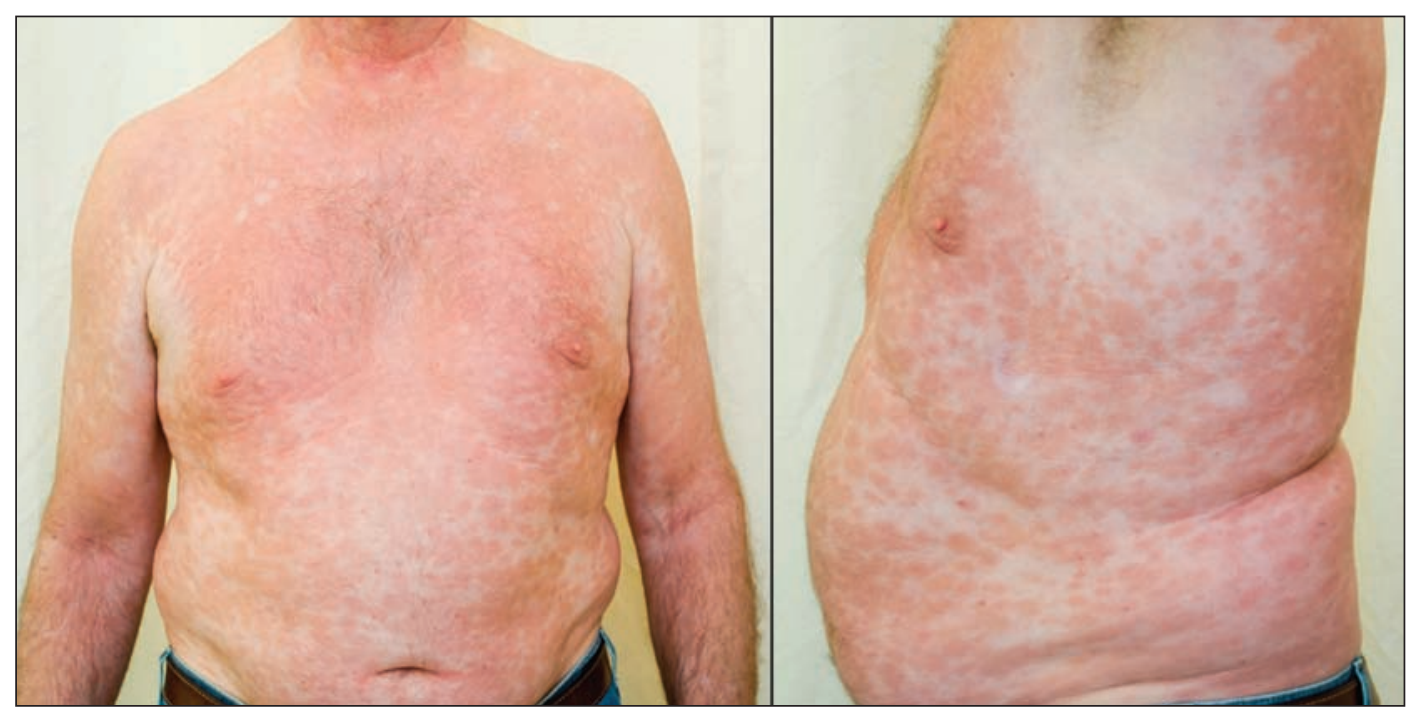

Figure 1: Confluent erythematous, maculopapular, hypoesthetic rash over the trunk and arms of a 60-yearold man with polyarthritis and neuropathy; the armpits, where the skin is warmer, are spared. 
His fingers were swollen, and he had many lacerations and burn marks on his hands and fingers (Figure 2). On neurologic examination, the cranial nerves were intact, but he had markedly decreased sensation in his hands, arms and feet. Pain (pinprick), temperature and vibration sensations were all affected. The triceps, brachioradialis and ankle jerk reflexes were absent. There was no palpable peripheral nerve enlargement, muscle weakness or fasciculation. Other findings of the neurologic examination were normal; in particular, there was neither adenopathy nor hepatosplenomegaly.

Laboratory data showed normal complete blood count, creatinine level, and liver enzyme levels and function. The erythrocyte sedimentation rate, C-reactive protein level, and antinuclear antibody, rheumatoid factor and antiCCP titres were also within normal limits. HIV antibody testing yielded negative results.

\section{What investigation(s) would you consider next?}
a) Antisynthetase antibody testing
b) Urine porphyrin test
c) Measurement of arsenic blood level
d) Skin biopsy

We measured the patient's blood arsenic level (c) and performed a skin biopsy (d). Chronic arsenic intoxication may present with peripheral neuropathy and hypopigmented or hyperpigmented skin

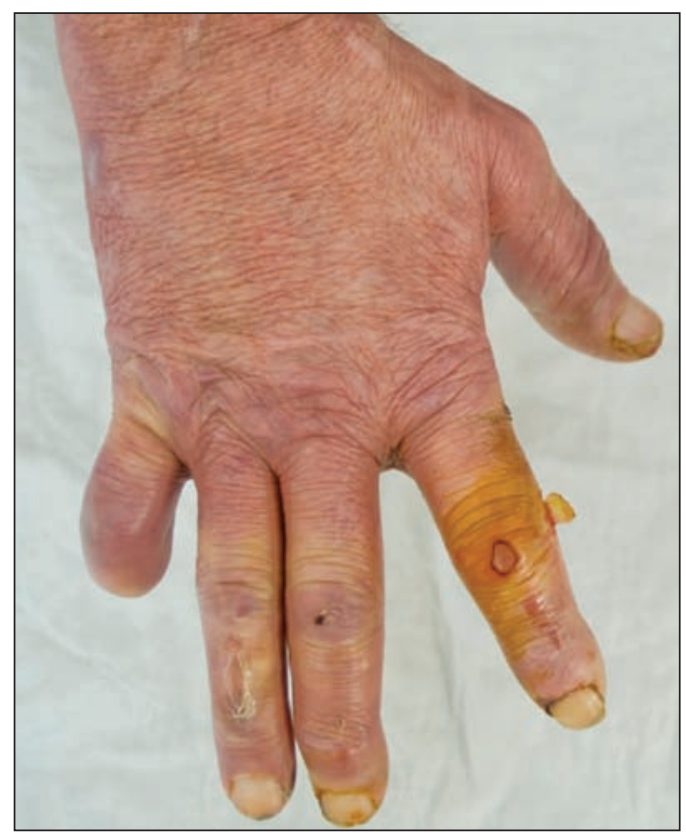

Figure 2: Swollen fingers and hand, with traumatic lacerations and burn marks on fingers. The amputation of the fifth finger was accidental and not related to the patient's pathology. lesions and cannot be excluded clinically, but arthritis is not a characteristic finding. Our patient had no history of arsenic exposure, and the blood arsenic level was within normal limits. With chronic arsenic intoxication ruled out, lepromatous leprosy became the most likely diagnosis. Moreover, leprosy could explain the patient's arthritis and hypogonadism. However, hypoesthesia is not typically found in the diffuse skin lesions seen in lepromatous leprosy, whereas tuberculoid leprosy usually presents with one or several hypoesthetic lesions. We also considered porphyria cutanea tarda, systemic lupus erythematosus, cutaneous lymphoma and dermatomyositis as possible diagnoses, which can present with similar skin lesions; however, peripheral neuropathy is not a typical clinical feature of these conditions.

The skin biopsy showed lymphohistiocytic inflammatory infiltrate, as well as many acid-fast bacilli, which were also detected with the use of Ziehl-Neelsen and auramine staining techniques (Figure 3). The bacterial index for leprosy was 4+ (10-100 bacilli per microscopic field). This finding was consistent with lepromatous leprosy. The patient later confirmed that he had travelled several times to Haiti and the Philippines, where the disease is endemic. He had close contact with people living in these areas. Most of his stays in these countries were less than four weeks.

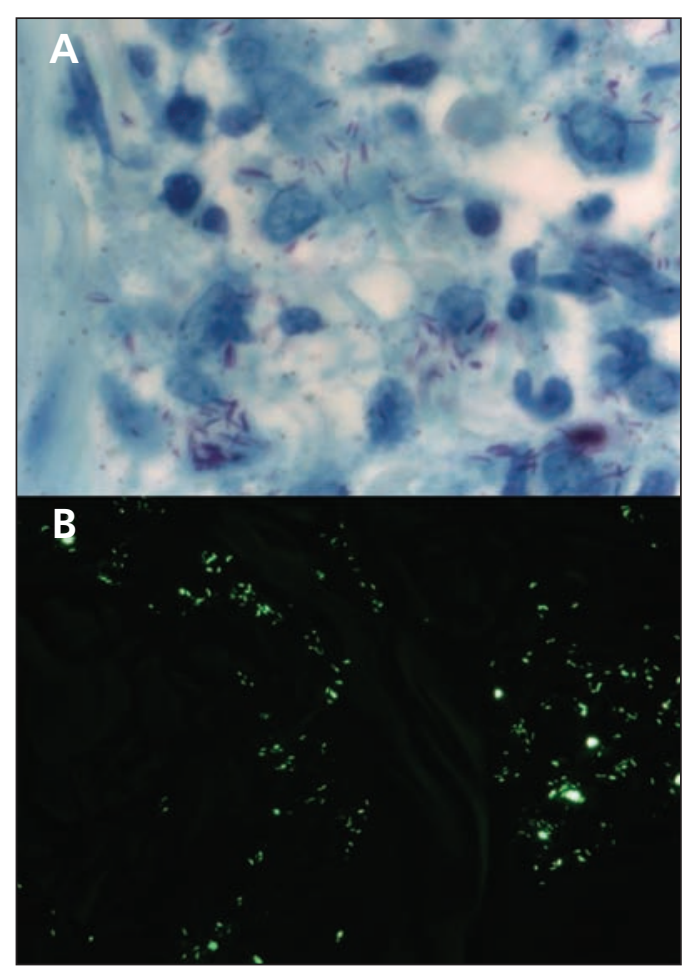

Figure 3: Full-thickness skin biopsy specimen, showing the presence of acid-fast bacilli with (A) ZiehlNeelsen stain (magnification $\times 1000$ ) and (B) auramine stain (magnification $\times 400$ ), findings that are consistent with a diagnosis of leprosy. 


\section{What tests are required to confirm the diagnosis?}

a) No further testing is required

b) Polymerase chain reaction for Mycobacterium leprae

c) Culture of tissue samples

d) Serologic testing for M. leprae

With atypical clinical features, as in our patient's case, the diagnosis of leprosy can be confirmed by means of polymerase chain reaction for M. leprae (b), the results of which were positive in our patient. Because M. leprae does not grow on culture media used in clinical laboratories, culture of tissue samples is not useful. Various serologic tests are available, but none is sufficiently specific or sensitive for leprosy.

The patient was prescribed dapsone $100 \mathrm{mg} / \mathrm{d}$, rifampin $600 \mathrm{mg} / \mathrm{d}$ and clofazimine $50 \mathrm{mg} / \mathrm{d}$. The treatment course was 24 months. A few months after starting the treatment, the patient noticed a substantial improvement in his arthritis and rash, as well as a thinning of the skin over his nose and decreased nasal congestion. Despite two years of treatment, the peripheral neuropathy did not improve and remained disabling. The arthritis, however, subsided permanently, confirming that it was associated with the leprosy. No immunemediated reaction occurred during treatment. Thereafter, the patient had a typical type 2 reversal reaction (erythema leprosum nodosum) but responded well to low-dose thalidomide treatment.

\section{Discussion}

In Canada, leprosy remains a rare disease, and most cases originate from immigration. ${ }^{1}$ The prevalence of leprosy in Canada is estimated to be 0.6 per 100000 population. ${ }^{2}$ Worldwide, 212000 new cases were reported in 2010, mainly in India, Brazil, the Democratic Republic of Congo and Indonesia. ${ }^{3}$ The incubation period from infection to development of the disease varies from a few months to 30 years; therefore, patients may present with leprosy several years after leaving a diseaseendemic region. Travel-associated leprosy is rare.

\section{Spectrum of the disease}

The World Health Organization (WHO) proposed a simplified classification of the disease according to the number of skin lesions: paucibacillary leprosy (which includes tuberculoid and borderline tuberculoid leprosy) and multibacillary leprosy (which includes midborderline, borderline lepromatous and lepromatous leprosy). ${ }^{4}$ At the paucibacillary tuberculoid pole of the disease spectrum, patients can have one or several macules or plaques with well-defined borders and hypopigmented, hypoesthetic centres. Enlargement of peripheral nerves, with or without tenderness, and marked nerve damage are common, often resulting in muscle weakness or trauma secondary to sensory loss.

Patients at the lepromatous pole of the disease spectrum present with symmetrically disseminated hypopigmented or erythematous skin lesions (macules, papules or nodules). Affected areas, including eyebrows and eyelashes, are hairless, but scalp hair is usually spared. Small dermal sensory nerves are affected, which results in glove-stocking sensory loss. As the disease progresses, the skin thickens, predominantly in the forehead, eyebrows and cheeks, which gives rise to the classic leonine facies. ${ }^{5}$ We hypothesized that, given the presence of hypoesthetic macules, our patient originally had a tuberculoid presentation that was pushed toward lepromatous leprosy by the immunosuppressive medication. Box 1 summarizes the typical clinical manifestations seen in leprosy. ${ }^{5-9}$

\section{Making the diagnosis}

In disease-endemic countries, classic clinical signs can be enough to make the diagnosis of leprosy. Elsewhere, the diagnosis is often delayed because of lack of clinical suspicion and unfamiliarity with the disease. A study involving 28 people with leprosy in a tropical diseases hospital in the United Kingdom showed that the mean time from onset of symptoms to diagnosis was about three years. ${ }^{10}$

A clinical presentation of leprosy with a predominance of extracutaneous symptoms, as seen in our patient, is challenging for clinicians.

Box 1: Clinical manifestations of leprosy *5-9

Dermal system (typically affected)

- Hypopigmented or erythematous macules, papules or plaques

- Leonine facies

- Hair loss, including eyebrows and eyelashes

Neurologic system (typically affected)

- Mononeuritis

- Mononeuritis multiplex

- Peripheral neuropathy

Musculoskeletal system

- Polyarthritis (44\%)

- Oligoarthritis (10\%)

- Neuropathic arthropathy (10\%)

Ophthalmic system

- Lagophthalmos (3\%-4\%)

- Corneal hypoesthesia (13\%)

- Cataract $(10 \%)$

- Blindness (3\%-7\%)

Endocrine system

- Hypogonadism (8\%-80\%)

*Percentages represent approximate proportion of patients affected. 
Involvement of the musculoskeletal system is the third most common manifestation of leprosy, but it is underrecognized. (The most common manifestations are dermal and sensory changes.) Acute polyarthritis in leprosy is symmetrical and affects mainly small joints of the hands and feet. A chronic, symmetrical, rheumatoid-like arthritis with insidious onset and morning stiffness, affecting primarily the wrists, metacarpophalangeal joints, proximal interphalangeal joints, knees and metatarsophalangeal joints has been described. ${ }^{6,11}$ Joint erosion and deformities similar to rheumatoid arthritis are also possible. Patients with leprosy may be positive for rheumatoid factor, which can further complicate the evaluation of arthritis.

Eye complications in leprosy are frequent, and up to $11 \%$ of patients with the disease may become blind. ${ }^{7}$ Eye complications occur usually in older people with long-standing multibacillary leprosy. Eye lesions may progress over years, even after patients have completed their treatment. ${ }^{8}$ Hypogonadism may also occur in leprosy, the frequency ranging from $8 \%$ to $80 \% .^{12}$

It can sometimes be difficult to detect acid-fast bacilli with histopathologic methods in early stages of the disease. New diagnostic tools are needed to increase the speed and accuracy of the diagnosis. Polymerase chain reaction can detect small quantities of bacilli in tissues. In countries where leprosy is not endemic, it is used primarily for the definitive diagnosis of $M$. leprae infection when acid-fast bacilli are observed, but atypical clinical or histopathologic features obscure the diagnosis. ${ }^{13}$ In a study involving 3525 patients with leprosy referred to the US National Hansen's Disease Program between 1993 and 2002, polymerase chain reaction had an overall sensitivity of $60 \%$ and a specificity of $100 \%$; its sensitivity for the lepromatous leprosy subgroup was $93 \% .^{14}$

\section{Treatment options}

Multidrug therapy is the standard treatment of leprosy. The regimen recommended by the WHO for multibacillary leprosy is dapsone $100 \mathrm{mg} / \mathrm{d}$, rifampin $600 \mathrm{mg}$ once a month and clofazimine $300 \mathrm{mg}$ once a month for 12 months. ${ }^{4}$ However, in developed countries, the National Hansen's Disease Program recommends daily (rather than monthly) doses of rifampin $600 \mathrm{mg}$ and clofazimime $50 \mathrm{mg}$ for 24 months. ${ }^{13}$ The difference between the two regimens is due largely to the WHO's cost considerations for developing countries where leprosy is endemic. In addition, there is some concern that the WHO treatment regimen may not be sufficient in patients who have a higher bacterial index. ${ }^{13}$ Even with treatment, morbidity secondary to nerve damage remains important and can be disabling. Up to $50 \%$ of patients with advanced lepromatous lep- rosy have permanent nerve damage.${ }^{15}$ Furthermore, nerve damage may be exacerbated by an immunemediated reaction during treatment.

\section{Immune-mediated reactions to treatment}

Two types of immune-mediated reactions can complicate treatment and occur in $20 \%-50 \%$ of patients with multibacillary leprosy. ${ }^{15}$ Type 1 or reversal reactions are characterized by fever, acute neuritis and inflamed skin lesions. Loss of nerve function can be substantial. Type 2 or erythema nodosum leprosum reactions are characterized by fever, arthritis, neuritis, orchitis, edema and erythema nodosum leprosum. Both types are more common during the first year of treatment.

Immune-mediated reactions are difficult to manage and need to be controlled, sometimes on an emergency basis, with immunosuppressive and immunomodulatory medications, such as prednisone, cyclosporine, azathioprine or methotrexate. ${ }^{15}$ For this reason, treatment should be prescribed and monitored by a clinician who is experienced in the treatment of leprosy.

\section{References}

1. Boggild AK, Correia JD, Keystone JS, et al. Leprosy in Toronto: an analysis of 184 imported cases. CMAJ 2004;170:55-9.

2. World Health Organization. Leprosy, global situation. Wkly Epidemiol Rec 2002;77:1-8.

3. World Health Organization. Global leprosy situation, 2010. Wkly Epidemiol Rec 2010;85:337-48.

4. WHO Expert Committee on Leprosy. 7th report [Technical Report Series, no. 874]. Geneva (Switzerland): World Health Organization; 1998.

5. Boggild AK, Keystone JS, Kain KC. Leprosy: a primer for Canadian physicians. CMAJ 2004;170:71-8.

6. Vengadakrishnan K, Saraswat PK, Mathur PC. A study of rheumatological manifestations of leprosy. Indian J Dermatol Venereol Leprol 2004;70:76-8.

7. Courtright P, Daniel E, Rao PS, et al. Eye disease in multibacillary leprosy patients at time of their leprosy diagnosis: findings from Longitudinal Study of Ocular Leprosy (LOSOL) in India, the Philippines and Ethiopia. Lepr Rev 2002;73:225-38.

8. Lewallen S, Tungpakkorn NC, Kim SH, et al. Progression of eye disease in "cured" leprosy patients: implications for understanding the pathophysiology of ocular disease and for addressing eyecare needs. Br J Ophthalmol 2000;84:817-21.

9. Chauhan S, Wakhlu A, Agarwal V. Arthritis in leprosy. Rheumatology 2010;49:2237-42.

10. Lockwood DNJ, Reid AJC. The diagnosis of leprosy is delayed in the United Kingdom. Q JM 2001;94:207-12.

11. Yens DA, Aster DJ, Teital A. Subcutaneous nodules and joint deformity in leprosy. J Clin Rheumatol 2003;9:181-6.

12. Leal AM, Foss NT. Endocrine dysfunction in leprosy. Eur J Clin Microbiol Infect Dis 2009;28:1-7.

13. National Hansen's Disease Center recommended treatment regimens. Rockville (MD): US Department of Health and Human Services, Health and Services Administration. Available: www.hrsa.gov/hansensdisease/diagnosis/recommendedtreatment .html (accessed 2013 Oct. 6).

14. Williams DL, Scollard DM, Gillis TP. PCR-based diagnosis of leprosy in the United States. Clin Microbiol Newsl 2003;25:57-61.

15. Rodrigues LC, Lockwood DN. Leprosy now: epidemiology, progress, challenges, and research gaps. Lancet Infect Dis 2011; 11:464-70.

Affiliations: Department of Medicine, Hôpital du SacréCoeur de Montréal, and Faculty of Medicine, University of Montréal, Montréal, Que.

Contributors: Both authors contributed substantially to the writing and revising of the article and gave final approval of the version submitted for publication. 